\title{
The quasiinvariants of the symmetric group
}

\author{
Jason Bandlow $\|$ and Gregg Musiker"非 \\ ${ }^{1}$ Department of Mathematics, University of California, One Shields Avenue, Davis, CA 95616, U.S.A. \\ ${ }^{2}$ Department of Mathematics, Massachusetts Institute of Technology, 77 Massachusetts Avenue, Cambridge, MA \\ 02139, U.S.A.
}

\begin{abstract}
For $m$ a non-negative integer and $G$ a Coxeter group, we denote by $\mathbf{Q} \mathbf{I}_{\mathbf{m}}(G)$ the ring of $m$-quasiinvariants of $G$, as defined by Chalykh, Feigin, and Veselov. These form a nested series of rings, with $\mathbf{Q I}_{\mathbf{0}}(G)$ the whole polynomial ring, and the limit $\mathbf{Q} \mathbf{I}_{\infty}(G)$ the usual ring of invariants. Remarkably, the ring $\mathbf{Q} \mathbf{I}_{\mathbf{m}}(G)$ is freely generated over the ideal generated by the invariants of $G$ without constant term, and the quotient is isomorphic to the left regular representation of $G$. However, even in the case of the symmetric group, no basis for $\mathbf{Q} \mathbf{I}_{\mathbf{m}}(G)$ is known. We provide a new description of $\mathbf{Q} \mathbf{I}_{\mathbf{m}}\left(S_{n}\right)$, and use this to give a basis for the isotypic component of $\mathbf{Q} \mathbf{I}_{\mathbf{m}}\left(S_{n}\right)$ indexed by the shape $[n-1,1]$.

Résumé. Pour $m$ un entier positif ou nul et $G$ un groupe de Coxeter, nous notons $Q I_{m}(G)$ l'anneau des quasiinvariants définis par Chalykh, Feigin et Veselov. On obtient ainsi une série d'anneaux emboités, $Q I_{0}(G)$ étant l'anneau des polynômes, et la limite $Q I_{\infty}(G)$ l'anneau des invariants usuels. Il est remarquable que l'anneau $Q I_{m}(G)$ est librement généré sur l'idéal engendré par les invariants de $G$ sans terme constant, et le quotient est isomorphe à la représentation régulière à gauche de $G$. Cependant, même dans le cas du groupe symétrique, aucune base de $Q I_{m}(G)$ n'est connue. Nous donnons une nouvelle description de $Q I_{m}(G)$ et l'utilisons pour obtenir une base du composant isotypique de $Q I_{m}\left(S_{n}\right)$ indexée par la partition $(n-1,1)$.
\end{abstract}

Keywords: symmetric group, invariants, quasiinvariants

\section{Introduction}

A permutation $\sigma \in S_{n}$ acts on a polynomial in $\mathbf{R}=\mathbb{Q}\left[x_{1}, \ldots, x_{n}\right]$ by permutation of indices:

$$
\sigma P\left(x_{1}, \ldots, x_{n}\right)=P\left(x_{\sigma(1)}, \ldots, x_{\sigma(n)}\right) .
$$

The $S_{n}$-invariant polynomials are known as symmetric functions, and denoted by $\Lambda_{n}$. It is well known (e.g., [Sta99]) that the elements of $\Lambda_{n}$ without constant term are generated by the elementary symmetric functions $\left\{e_{1}, \ldots, e_{n}\right\}$ where

$$
e_{j}=\sum_{i_{1}<i_{2}<\cdots<i_{j}} x_{i_{1}} \ldots x_{i_{j}}
$$

\footnotetext{
${ }^{\dagger} \mathrm{NSF}$ grant DMS-0500557

$\ddagger$ NSF grant DMS-0500557
} 
The ring of coinvariants of $S_{n}$ is the quotient

$$
\mathbf{R} /\left\langle e_{1}, \ldots, e_{n}\right\rangle
$$

As an $S_{n}$-module, the ring of coinvariants is known to be isomorphic to the left regular representation. It is also known that $\mathbf{R}$ is freely generated over this ideal which implies that if we choose a basis of minimal degree elements $\mathcal{B}=\left\{b_{1}, \ldots, b_{n}\right.$ ! $\}$ for the ring of coinvariants, any element $P \in \mathbf{R}$ has a unique expansion

$$
P=\sum_{i=1}^{n !} b_{i} g_{i}
$$

with the $g_{i} \in \Lambda_{n}$. More information is given by the Hilbert series for the isotypic component of $\mathbf{R}$ corresponding to $\lambda$, namely

$$
\frac{\sum_{T \in S T(\lambda)} f_{\lambda} q^{\operatorname{cocharge}(T)}}{(1-q)\left(1-q^{2}\right) \ldots\left(1-q^{n}\right)} .
$$

Known bases for the ring of coinvariants with combinatorial descriptions include the Artin monomials and the Schubert polynomials.

In [CV90] and [FV02], Chalykh, Feigin and Veselov introduced a generalization of invariance known as " $m$-quasiinvariance". For the symmetric group, the $m$-quasiinvariants are the polynomials $P \in$ $\mathbb{Q}\left[x_{1}, \ldots, x_{n}\right]$ which have the divisibility property

$$
\left(x_{i}-x_{j}\right)^{2 m+1} \mid(1-(i, j)) P
$$

for every transposition $(i, j)$. We set

$$
\mathbf{Q I}_{m}=\left\{m \text {-quasiinvariants of } S_{n}\right\} \text {. }
$$

A straightforward calculation (which we do not do here) shows that the $m$-quasiinvariants of $S_{n}$ form a ring and an $S_{n}$ module, and that we have the following containments:

$$
\mathbf{R}=\mathbf{Q I}_{0} \supset \mathbf{Q I}_{1} \supset \cdots \supset \mathbf{Q I}_{m} \supset \cdots \supset \Lambda_{n}
$$

For all $m$, the ring $\mathbf{Q} \mathbf{I}_{m}$ was conjectured in [FV02] to be freely generated over the ideal generated by symmetric functions without constant term. The corresponding quotient $\mathbf{Q I}_{m} /\left\langle e_{1}, \ldots, e_{n}\right\rangle$ was conjectured to be isomorphic as an $S_{n}$ module to the left regular representation. These facts were proved in [EG02]. Further information was given in [FV03], where they showed the Hilbert series of the isotypic component of $\mathbf{Q I} \mathbf{I}_{m}$ indexed by $\lambda$ is given by

$$
\frac{\sum_{T \in S T(\lambda)} f_{\lambda} q^{m\left(\left(\begin{array}{l}
n \\
2
\end{array}\right)-\operatorname{content}(\lambda(T))\right)+\operatorname{cocharge}(T)}}{(1-q)\left(1-q^{2}\right) \ldots\left(1-q^{n}\right)} .
$$

Here $f_{\lambda}$ is the number of standard tableaux of shape $\lambda$ and content and cocharge are two statistics on tableaux-we will not need the precise definitions, though we note that content only depends on the 
shape of $T$ and thus is actually a statistic on partitions. We should note that the definitions, theorems and conjectures cited above were all phrased in terms of general Coxeter groups; it is only for simplicity that we have restricted our attention to $S_{n}$.

In light of the combinatorially interesting bases for the coinvariants in the classical (i.e., $m=0$ ) case, the authors have looked for a basis for larger $m$. In [FV02], and later in [BM05], a basis was given for the case $n=3$. (The work [FV02] specifically described the quasiinvariants for dihedral groups; in particular for $D_{3} \cong S_{3}$.) Further, in [FV03], Felder and Veselov provide integral expressions, $\phi^{(j)}(x)$ for $2 \leq j \leq n$, for the lowest degree non-symmetric $m$-quasiinvariants, i.e. those of degree $m n+1$. In the present work, we give a complete basis of the isotypic component indexed by the partition $[n-1,1]$. This is accomplished by means of a new characterization of $\mathbf{Q} \mathbf{I}_{m}$ :

Theorem 1 The vector space of quasiinvariants has the following direct sum decomposition:

$$
\mathbf{Q} \mathbf{I}_{m}=\bigoplus_{T \in S T(n)}\left(\gamma_{T} \mathbf{R} \cap V_{T}^{2 m+1} \mathbf{R}\right)
$$

where $S T(n)$ is the set of standard tableaux of size $n, \gamma_{T}$ is a projection operator due to Young (defined in full detail in the next section) and $V_{T}$ is the polynomial given by the product over the columns of $T$ of the associated "Vandermonde determinants" (this is also defined in detail below). This characterization is proved using completely elementary methods. We then give a basis for the $[n-1,1]$ isotypic component, using the previous characterization to show that our basis does, in fact, consist of quasiinvariants. Precisely, for $T$ a standard Young tableau of shape $[n-1,1]$ with $j$ the entry in the second row, we set

$$
Q_{T}^{k, m}=\int_{x_{1}}^{x_{j}} t^{k} \prod_{i=1}^{n}\left(t-x_{i}\right)^{m} d t .
$$

With this definition, we have

Theorem 2 The set

$$
\left\{Q_{T}^{0, m}, Q_{T}^{1, m}, Q_{T}^{2, m}, \ldots, Q_{T}^{n-2, m}\right\}
$$

is a basis for $\gamma_{T}\left(\mathbf{Q I}_{m} /\left\langle e_{1}, \ldots, e_{n}\right\rangle\right)$.

The fact $\mathbf{Q} \mathbf{I}_{m}$ is freely generated over $\left\langle e_{1}, \ldots, e_{n}\right\rangle$ then gives us that the set

$$
\left\{Q_{T}^{k, m} e_{1}^{p_{1}} e_{2}^{p_{2}} \ldots e_{n}^{p_{n}}\right\} \quad 0 \leq k \leq n-2, \quad p_{i} \in \mathbb{N}
$$

is a basis for $\mathbf{Q} \mathbf{I}_{m}$.

\section{Acknowledgements}

The authors are grateful for Adriano Garsia's guidance in this project, as well as for the support of the NSF. 


\section{Definitions and notation}

Throughout this work, we will write elements of the symmetric group $S_{n}$ using cycle notation. We will perform computations in the group algebra of $S_{n}$, and as such it will be useful to have shorthand notation for many commonly occurring elements. For a given subgroup $A$ of $S_{n}$, we set

$$
\begin{aligned}
& {[A]=\sum_{\sigma \in A} \sigma \quad \text { and }} \\
& {[A]^{\prime}=\sum_{\sigma \in A} \operatorname{sgn}(\sigma) \sigma .}
\end{aligned}
$$

The Young diagram of a partition $\lambda$ consists of a collection of $\lambda_{i}$ left-justified boxes in the $i^{t h}$ row of the positive integer lattice. These boxes are indexed by ordered pairs $(i, j)$, where $i$ is the row index and $j$ is the column index. For example, in the following Young diagram of $[4,3,2]$, the cell $(2,3)$ is marked:

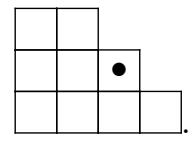

A tableau of shape $\lambda \vdash n$ is a function from the cells of the Young diagram of $\lambda$ to the set $\{1, \ldots, n\}$. We write the $T(i, j)$ for the value of $T$ at the cell $(i, j)$. For example, if $T$ is the following tableau, $T(2,3)=8$ :

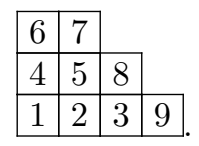

We call a tableau standard if it is injective and the entries increase across the rows and up the columns. For example, the tableau above is standard. We denote the set of standard tableaux of shape $\lambda$ by $S T(\lambda)$ and the set of all standard tableaux with $n$ boxes by $S T(n)$.

Given a tableau $T$ we let $C_{i}$ be the set of elements in the $i^{t h}$ column and we define $R_{i}$ similarly for the rows. We also set

$$
\begin{aligned}
C(T) & =\bigcup_{i}\left\{(a, b): a, b \in C_{i}\right\} \\
N(T) & =\prod_{i}\left[C_{i}\right]^{\prime} \\
P(T) & =\prod_{i}\left[R_{i}\right] \\
\gamma_{T} & =\frac{f_{\lambda} N(T) P(T)}{n !} \\
\lambda(T) & =\text { the shape of tableau } T .
\end{aligned}
$$

Finally, we define the following useful polynomial associated with a tableau $T$ :

$$
V_{T}=\prod_{(i, j) \in C(T)}\left(x_{i}-x_{j}\right) .
$$




\section{Useful Facts From Representation Theory}

The following is a fundamental fact about the representation theory of the symmetric group.

Proposition 1 For $W$ a finite dimensional $S_{n}$-module,

$$
W \cong \bigoplus_{\lambda \vdash n} V_{\lambda}^{\oplus m_{\lambda}}
$$

where the $V_{\lambda}$ are the irreducible representations of $S_{n}$ and the $m_{\lambda}$ are non-negative integers.

The vector space and $S_{n}$-module $V_{\lambda}^{\oplus m_{\lambda}}$ is known as the isotypic component of $V$ indexed by $\lambda$. Now, $\mathbf{Q I}_{m}$ is infinite dimensional, but it is the direct sum of homogeneous components, each of which are finite dimensional. So we have that each homogeneous component of $\mathbf{Q \mathbf { I } _ { m }}$ decomposes into the direct sum of irreducibles. The direct sum of all copies of $V_{\lambda}$ occurring in this decomposition is still itself an $S_{n}$-module, and is still referred to as the isotypic component indexed by $\lambda$. However, we will find the following decomposition of $V$ more useful.

Proposition 2 On any $S_{n}$ module $W$, the group algebra elements $\left\{\gamma_{T}\right\}_{T \in S T(n)}$ act as projection operators. In symbols, we have the conditions

1. $\gamma_{T}^{2}=\gamma_{T}$

2. $W=\bigoplus_{T \in S T(n)} \gamma_{T} W$.

Note that in this decomposition, unlike the previous one, the direct summands are not themselves $S_{n^{-}}$ modules. We do have the following proposition, however, nicely relating the previous two.

Proposition 3 For any $S_{n}$ module $W$,

$$
\bigoplus_{T \in S T(\lambda)} \gamma_{T} W
$$

is the isotypic component of $W$ indexed by $\lambda$.

In the case of the quasiinvariants, we have the following

Proposition 4 The $\mathbb{Q}$-vector space of m-quasiinvariants has the following direct sum decomposition:

$$
\mathbf{Q I}_{m}=\bigoplus_{T \in S T(n)} \gamma_{T} \mathbf{Q I}_{m} .
$$

Our goal will be to use the decomposition $\mathbf{Q} \mathbf{I}_{m} /\left\langle e_{1}, \ldots e_{n}\right\rangle=\bigoplus_{T} \gamma_{T}\left(\mathbf{Q I}_{m} /\left\langle e_{1}, \ldots e_{n}\right\rangle\right)$ to find a partial basis for this space.

\section{A New Characterization of $S_{n}$-Quasiinvariants}

In this section we prove the following theorem:

Theorem 1 The vector space of quasiinvariants has the following direct sum decomposition:

$$
\mathbf{Q I}_{m}=\bigoplus_{T \in S T(n)}\left(\gamma_{T} \mathbf{R} \cap V_{T}^{2 m+1} \mathbf{R}\right) .
$$


We will prove this by showing

$$
\gamma_{T} \mathbf{Q I} \mathbf{I}_{m}=\gamma_{T} \mathbf{R} \cap V_{T}^{2 m+1} \mathbf{R} .
$$

Combining (20) with Proposition 4 will prove the theorem. We note first that one containment of 20$]$ is easy:

$$
\gamma_{T} \mathbf{Q I} \mathbf{I}_{m} \subseteq \gamma_{T} \mathbf{R} \cap V_{T}^{2 m+1} \mathbf{R}
$$

By definition, any element of $\gamma_{T} \mathbf{Q} \mathbf{I}_{m}$ is also in $\gamma_{T} \mathbf{R}$. Thus, we must only show that for $Q \in \mathbf{Q I} \mathbf{I}_{m}$ we have

$$
\gamma_{T} Q \in V_{T}^{2 m+1} \mathbf{R}
$$

Let $P=\gamma_{T} Q=N(T) Q^{\prime}$. P must be anti-symmetric with respect to all transpositions in $C(T)$ since it is in the image of $N(T)$. Thus, for any $(a, b) \in C(T),(1-(a, b)) P=2 P$. Hence $\left(x_{a}-x_{b}\right)^{2 m+1}$ divides $2 P$ (and also $P$ ) for all $(a, b) \in C(T)$. This establishes equation (21).

It remains to show that for all standard tableaux $T$ and all $m \geq 0$, we have the following containment of vector spaces:

$$
\gamma_{T} \mathbf{R} \cap V_{T}^{2 m+1} \mathbf{R} \subseteq \gamma_{T} \mathbf{Q} \mathbf{I}_{m}
$$

Since $\gamma_{T}$ is an idempotent, it suffices to show that for any polynomial $P$ in the ideal $V_{T}^{2 m+1} \mathbf{R}, \gamma_{T} P=$ $P$ implies that $P$ is $m$-quasiinvariant. With this in mind, we let $P$ be such that $V_{T}^{2 m+1} \mid P$ and $\gamma_{T} P=P$. We wish to show that $(1-(a, b)) P$ is divisible by $\left(x_{a}-x_{b}\right)^{2 m+1}$ for all transpositions $(a, b)$. We first consider the case where $a$ and $b$ are in the same column of $T$. In this case we have

$$
(a, b) N(T)=-N(T)
$$

and so

$$
(a, b) P=(a, b) \gamma_{T} P=-\gamma_{T} P=-P
$$

Thus

$$
(1-(a, b)) P=2 P \in V_{T}^{2 m+1} \mathbf{R}
$$

which is divisible by the required factor of $\left(x_{a}-x_{b}\right)^{2 m+1}$.

The remaining case, showing that $(1-(a, b)) P$ is divisible by $\left(x_{a}-x_{b}\right)^{2 m+1}$ when $a$ and $b$ are in different columns, is the only difficult part of the proof. Suppose without loss that $a$ is in column $i$, and is to the left of $b$, which is in column $j$. We define $\alpha_{i, b} \in \mathbb{Q}\left[S_{n}\right]$ to be the sum of all transpositions $(c, b)$ where $c$ is an element of column $i$. The key property of this is element is the following: 
Lemma 1 The operator $\alpha_{i, b}$ preserves $\gamma_{T}$. In symbols,

$$
\alpha_{i, b} \gamma_{T}=\gamma_{T} .
$$

The proof of Lemma 1 though completely elementary, is lengthy and is omitted here.

With this fact in hand, it is not difficult to complete the proof. By Lemma 1, $P$ is preserved by $\alpha_{i, b}$ :

$$
\alpha_{i, b} P=\alpha_{i, b} \gamma_{T} P=\gamma_{T} P=P .
$$

This fact and the definition of $\alpha_{i, b}$ gives

$$
\begin{aligned}
(1-(a, b)) P & =P-(a, b) P \\
& =\alpha_{i, b} P-(a, b) P \\
& =\sum_{\substack{c \in C_{i} \\
c \neq a}}(c, b) P .
\end{aligned}
$$

Since $P \in V_{T}^{2 m+1} \mathbf{R}$, for any $c \in C_{i}$ with $c \neq a$ we can rewrite $P$ as

$$
P=\left(x_{c}-x_{a}\right)^{2 m+1} \text { (other factors). }
$$

Thus

$$
(c, b) P=\left(x_{b}-x_{a}\right)^{2 m+1}(\text { other factors })
$$

and we have

$$
\left(x_{b}-x_{a}\right)^{2 m+1} \text { divides }(c, b) P \text { for every } c \in C_{i} \text { with } c \neq a .
$$

Hence $\left(x_{b}-x_{a}\right)^{2 m+1}$ divides the right-hand side of equation 27, which completes the proof.

\section{$6 \quad$ A Basis For The Isotypic Component $\lambda(T)=[n-1,1]$}

In this section, we refer to the quotient $\mathbf{Q I} \mathbf{I}_{m} /\left\langle e_{1}, \ldots, e_{n}\right\rangle$ by the symbol $\mathbf{Q I}_{m}^{*}$. Our object here is to describe a basis for $\gamma_{T} \mathbf{Q} \mathbf{I}_{m}^{*}$ when $T$ has a hook shape of the form $[n-1,1]$. Until otherwise specified, let $\lambda$ be the partition $[n-1,1]$ and let $T$ be one of the $(n-1)$ standard tableaux of shape $\lambda$. In fact $T$ is uniquely defined by the lone entry of the second row. Suppose it's $j \in\{2,3, \ldots, n\}$. We define

$$
Q_{T}^{k, m}=\int_{x_{1}}^{x_{j}} t^{k} \prod_{i=1}^{n}\left(t-x_{i}\right)^{m} d t .
$$

Theorem 2 The polynomials $\left\{Q_{T}^{k, m}\right\}_{k=0}^{n-2}$ are a set of representatives for a basis of $\gamma_{T} \mathbf{Q} \mathbf{I}_{m}^{*}$.

By equation (9) we know this consists of the right number of elements, and they are of the right degree. Thus we are faced with two tasks: showing the elements $Q_{T}^{k, m}$ are, in fact, $m$-quasiinvariant, and showing that they are linearly independent in $\mathbf{Q} \mathbf{I}_{m}^{*}$. To show the $m$-quasiinvariance of $Q_{T}^{k, m}$ we must, by Theorem 1 show these polynomials are preserved by $\gamma_{T}$ and divisible by $V_{T}^{2 m+1}$. 
Lemma 2 The polynomials $Q_{T}^{k, m}$ are preserved by the operator $\gamma_{T}$. That is,

$$
\gamma_{T} Q_{T}^{k, m}=Q_{T}^{k, m}
$$

Proof: We will prove this inductively, by showing that the $Q_{T}^{k, m}$ satisfy a recursion that respects the action of $\gamma_{T}$. In what follows, $e_{i}$ will denote the $i$ th elementary symmetric function in the variables $x_{1}, \ldots, x_{n}$, with the convention that $e_{0}=1$. We first state for reference a classical symmetric function identity:

$$
\prod_{i=1}^{n}\left(t-x_{i}\right)=\sum_{i=0}^{n}(-1)^{i} e_{i} t^{n-i}
$$

We now show that for $m>1$, we have the recurrence:

$$
Q_{T}^{k, m}=\sum_{i=0}^{n}(-1)^{i} e_{i} Q_{T}^{n-i+k, m-1}
$$

This can be seen by first expanding the definition of $Q_{T}^{k, m}$ to get

$$
Q_{T}^{k, m}=\int_{x_{1}}^{x_{j}}\left(\prod_{i=1}^{n}\left(t-x_{i}\right)\right) t^{k} \prod_{l=1}^{n}\left(t-x_{l}\right)^{m-1} d t .
$$

Substituting 33 into 35 and pulling out the factors not involving $t$ gives

$$
\begin{aligned}
Q_{T}^{k, m} & =\int_{x_{1}}^{x_{j}}\left(\sum_{i=0}^{n}(-1)^{i} e_{i} t^{n-i}\right) t^{k} \prod_{l=1}^{n}\left(t-x_{l}\right)^{m-1} d t \\
& =\sum_{i=0}^{n}(-1)^{i} e_{i} \int_{x_{1}}^{x_{j}} t^{n-i+k} \prod_{l=1}^{n}\left(t-x_{l}\right)^{m-1} d t \\
& =\sum_{i=0}^{n}(-1)^{i} e_{i} Q_{T}^{n-i+k, m-1} .
\end{aligned}
$$

We now proceed with a proof of Lemma 2 by induction on $m$. From the definition of $Q_{T}^{k, m}$ we have

$$
\begin{aligned}
Q_{T}^{k, 0} & =\int_{x_{1}}^{x_{j}} t^{k} \prod_{i=1}^{n}\left(t-x_{i}\right)^{0} d t \\
& =\frac{1}{k+1}\left(x_{j}^{k+1}-x_{1}^{k+1}\right) .
\end{aligned}
$$

That $x_{j}^{k+1}-x_{1}^{k+1}$ is preserved by $\gamma_{T}$ is a straightforward calculation which we omit here. 
We can now complete the proof by using equation (34), the inductive hypothesis, and the fact that symmetric functions commute with group algebra elements:

$$
\begin{aligned}
\gamma_{T} Q_{T}^{k, m} & =\sum_{i=0}^{n}(-1)^{i} \gamma_{T}\left(e_{i} Q_{T}^{n-i+k, m-1}\right) \\
& =\sum_{i=0}^{n}(-1)^{i} e_{i}\left(Q_{T}^{n-i+k, m-1}\right) \\
& =Q_{T}^{k, m} .
\end{aligned}
$$

In order to complete our task of showing that $Q_{T}^{k, m} \in \mathbf{Q I}_{m}$, we must show that $\left(x_{j}-x_{1}\right)^{2 m+1}$ divides $Q_{T}^{k, m}$. We do so by proving the following stronger statement:

Lemma 3 For all $k$,

$$
\lim _{x_{1} \rightarrow x_{j}} \frac{Q_{T}^{k, m}}{\left(x_{j}-x_{1}\right)^{2 m+1}}=\frac{(-1)^{m} m !^{2}}{(2 m+1) !} x_{j}^{k} \prod_{\substack{i=2 \\ i \neq j}}^{n}\left(x_{j}-x_{i}\right)^{m} .
$$

Proof: This is a straightforward calculation. Expanding $Q_{T}^{k, m}$ according to the definition gives

$$
\lim _{x_{j} \rightarrow x_{1}} \frac{Q_{T}^{k, m}}{\left(x_{j}-x_{1}\right)^{2 m+1}}=\lim _{x_{j} \rightarrow x_{1}} \frac{\int_{x_{1}}^{x_{j}} t^{k} \prod_{i=1}^{n}\left(t-x_{i}\right)^{m} d t}{\left(x_{j}-x_{1}\right)^{2 m+1}}
$$

which is an indeterminate expression of the form $\frac{0}{0}$. Repeatedly applying L'Hopital's rule and evaluating the numerator with Leibniz's rule for differentiation under the integral gives that the expression in (44) equals

$$
\lim _{x_{j} \rightarrow x_{1}} \frac{(-1)^{m} \cdot m ! \int_{x_{1}}^{x_{j}} t^{k} \prod_{\substack{i=1 \\ i \neq j}}^{n}\left(t-x_{i}\right)^{m} d t}{(2 m+1)(2 m)(2 m-1) \cdots(m+2)\left(x_{j}-x_{1}\right)^{m+1}} .
$$

One more application of L'Hopital's rule, evaluated this time with the Fundamental Theorem of Calculus, yields

$$
\lim _{x_{j} \rightarrow x_{1}} \frac{(-1)^{m} \cdot m ! \cdot x_{j}^{k} \prod_{\substack{i=1 \\ i \neq j}}^{n}\left(x_{j}-x_{i}\right)^{m}}{(2 m+1)(2 m)(2 m-1) \cdots(m+1)\left(x_{j}-x_{1}\right)^{m}} .
$$

We now cancel the term $\left(x_{j}-x_{1}\right)^{m}$ from both numerator and denominator and the Lemma is proven.

The polynomiality of $\lim _{x_{1} \rightarrow x_{j}} \frac{Q_{T}^{k, m}}{\left(x_{j}-x_{1}\right)^{2 m+1}}$ immediately gives that $\left(x_{j}-x_{1}\right)^{2 m+1} \operatorname{divides} Q_{T}^{k, m}$. Thus we have established that $Q_{T}^{k, m}$ is $m$-quasiinvariant.

We need one more lemma to establish Theorem 2 . 
Lemma 4 The set $\left\{Q_{T}^{k, m}\right\}_{k=0}^{n-2}$ is linearly independent in $\mathbf{Q \mathbf { I } _ { m } ^ { * }}$.

Proof: As these polynomials are of different degrees, it suffices to show that they are non-zero in $\mathbf{Q} \mathbf{I}_{m}^{*}$. Put another way, we must show that $Q_{T}^{k, m}$ is not in the ideal of $\gamma_{T} \mathbf{Q} \mathbf{I}_{m}$ generated by $\left\langle e_{1}, \ldots, e_{n}\right\rangle$. Equivalently, we must show that polynomials of the form

$$
P_{k}=Q_{T}^{k, m}+A_{1} Q_{T}^{k-1, m}+\cdots+A_{k-1} Q_{T}^{1, m}+A_{k} Q_{T}^{0, m}
$$

(where the $A_{i}$ are symmetric functions of degree $i$ ) can only equal 0 if $k \geq n-1$. To accomplish this, we use the explicit formulas for $\lim _{x_{j} \rightarrow x_{1}} Q_{T}^{k, m} / V_{T}^{2 m+1}$ given by Lemma 3 to show the stronger statement

$$
\lim _{x_{j} \rightarrow x_{1}} P_{k} / V_{T}^{2 m+1}=0 \Longrightarrow k \geq n-1
$$

regardless of the choice of the symmetric functions. Letting $\widetilde{A_{i}}$ denote the limit $x_{j} \rightarrow x_{1}$ applied to the symmetric function $A_{i}$, and assuming without loss of generality that $j=2$, we have

$$
\begin{aligned}
& \lim _{x_{2} \rightarrow x_{1}} P_{k} / V_{T}^{2 m+1}=0 \\
\Longrightarrow & \left(\frac{(-1)^{m} m !^{2}}{(2 m+1) !} \prod_{i=3}^{n}\left(x_{1}-x_{i}\right)^{m}\right)\left(x_{1}^{k}+\widetilde{A_{1}} x_{1}^{k-1}+\cdots+\widetilde{A_{k-1}} x_{1}+\widetilde{A_{k}}\right)=0 \\
\Longrightarrow & x_{1}^{k}+\widetilde{A_{1}} x_{1}^{k-1}+\cdots+\widetilde{A_{k-1}} x_{1}+\widetilde{A_{k}}=0 \\
\Longrightarrow & \lim _{x_{2} \rightarrow x_{1}}\left(x_{1}^{k}+A_{1} x_{1}^{k-1}+\cdots+A_{k}\right)=0 .
\end{aligned}
$$

We set

$$
Q\left(x_{1}, \ldots, x_{n}\right)=x_{1}^{k}+A_{1} x_{1}^{k-1}+\cdots+A_{k}
$$

and now equation 51 implies that

$$
Q\left(x_{1}, \ldots, x_{n}\right)=\left(x_{2}-x_{1}\right) \cdot R\left(x_{1}, \ldots, x_{n}\right) .
$$

However, $Q$ must be symmetric with respect to all pairs of variables not involving $x_{1}$. Thus, for any $\sigma \in S_{\{2,3, \ldots, n\}}, \sigma Q=Q$ and so

$$
Q\left(x_{1}, \ldots, x_{n}\right)=\sigma Q\left(x_{1}, \ldots, x_{n}\right)=\left(x_{\sigma(2)}-x_{1}\right) \cdot \sigma R\left(x_{1}, \ldots, x_{n}\right) .
$$

Hence $\prod_{i=2}^{n}\left(x_{i}-x_{1}\right)$ divides $Q\left(x_{1}, \ldots, x_{n}\right)$, and $k$, which is the degree of $Q\left(x_{1}, \ldots, x_{n}\right)$, must be greater than or equal to $n-1$.

The proof of Theorem 2 now follows immediately from Lemmas 2,3 , and 4 


\section{References}

[BM05] J. Bandlow and G. Musiker. Quasiinvariants of $S_{3}$. Journal of Comb. Theory, Ser. A, 109:281298, 2005.

[CV90] O. A. Chalykh and A. P. Veselov. Commutative rings of partial differential operators and Lie algebras. Commun. Math. Phys., (126):597-611, 1990.

[EG02] P. Etingof and V. Ginzburg. On $m$-quasi-invariants of a Coxeter group. Moscow Math. J., (3):555-566, 2002.

[FV02] M. Feigin and A. P. Veselov. Quasiinvariants of Coxeter groups and $m$-harmonic polynomials. Intern. Math. Res. Notices, (10):521-545, 2002.

[FV03] G. Felder and A. P. Veselov. Action of Coxeter groups on $m$-harmonic polynomials and Knizhnik-Zamolodchikov equations. Moscow Math. J., (4):1269-1291, 2003.

[Sta99] Richard P. Stanley. Enumerative Combinatorics, volume 2. Cambridge University Press, Cambridge, United Kingdom, 1999. 
